\title{
A Dual Spring Modeling Approach for Static and Fatigue Failure Assessments of Carbon/Epoxy Composite Sub- Elements
}

\author{
Xiaodong Cui ${ }^{1}$, Xiang Ren ${ }^{2}$, Phillip Liu ${ }^{3}, \mathrm{Jim} \mathrm{Lua}^{4}$ \\ Global Engineering and Materials, Inc., Princeton, NJ, 08540 \\ and \\ Prabhakar M. Rao ${ }^{5}$, Matthew D. Mordasky ${ }^{6}$, Mark R. Gurvich ${ }^{7}$ \\ United Technologies Research Center, East Harford, CT, 06108
}

\begin{abstract}
A dual spring model is developed for the static and fatigue damage predictions of laminates interface in composite structures. Stress concentrations can be induced by the defects formed in the fabrication or service process. A conventional $\mathrm{S}-\mathrm{N}$ based fatigue damage model may not be accurate to predict the fatigue life of a structure with high stress concentration. With the dual spring model, static delamination failure can be simulated using springs of cohesive type material model while fatigue delamination development can be predicted using linear springs, where the crack driving force is computed based on virtual crack closure technique (VCCT). A Paris law type fatigue growth law with its mode mixity is applied for fatigue crack growth prediction. After verified using benchmark examples, including Double Cantilever Beam (DCB), End-Notched Flexure (ENF) and Mix-Mode Bending (MMB), the proposed dual spring model is applied in the static and fatigue damage prediction of NASA/Boeing sub-elements and UTC sub-elements.
\end{abstract}

\section{Nomenclature}

$\begin{array}{ll}V C C T & =\text { virtual crack closure technique } \\ D C B & =\text { Double Cantilever Beam } \\ E N F & =\text { End-Notched Flexure } \\ M M B & =\text { Mix-Mode Bending } \\ B-K & =\text { Benzeggagh and Kenane } \\ G & =\text { strain energy release rate } \\ \eta & =\text { mode mixity parameter } \\ F_{P} & =\text { crack closing force } \\ \delta_{P} & =\text { opening displacement at sampling point } \\ A_{P} & =\text { nodal representative area } \\ c & =\text { Paris law coefficient } \\ m & =\text { Paris law power factor } \\ d a / d N & =\text { crack growth rate } \\ C D M & =\text { continuum damage mechanics }\end{array}$

\footnotetext{
${ }^{1}$ Senior Scientist, Global Engineering and Materials, Inc., Princeton, NJ 08540.

${ }^{2}$ Scientist, Global Engineering and Materials, Inc., Princeton, NJ 08540.

${ }^{3}$ Senior Scientist, Global Engineering and Materials, Inc., Princeton, NJ 08540.

${ }^{4}$ Senior Principal Scientist, Global Engineering and Materials, Inc., Princeton, NJ 08540, AIAA Member.

${ }^{5}$ Staff Engineer, Research, United Technologies Research Center, East Harford, CT 06108

${ }^{6}$ Staff Engineer, Research, United Technologies Research Center, East Harford, CT 06108

${ }^{7}$ Technical Fellow, Research, United Technologies Research Center, East Harford, CT 06108
} 


\section{Introduction}

$\mathrm{T}$ HE static and fatigue damage predictions of composite structures with the presence of stress concentrators are still a challenging task due to the existence of an inherent gap of a continuum damage mechanics and fracture mechanics based modeling approach. The presence of stress concentrations resulting from either the fabrication induced defects or the presence of geometric discontinuities can render the predictions of damage initiation mesh sensitive within a finite element solution framework. While an energy driven failure criterion can be included in the static damage prediction to alleviate the mesh dependence, its extension to fatigue damage initiation and propagation is not straight forward since the fatigue damage progression is controlled by the crack growth driving force along its front i.e. Paris law, without invoking an energy dissipation principle. A conventional S-N based fatigue damage initiation is applicable for composite components without the presence of stress singularity.

Both the cohesive ${ }^{1-4}$ and virtual crack closure technique ${ }^{5-8}$ (VCCT) have been used to perform fatigue damage evaluation of composite structures. The application of the cohesive model has shown its benefit when simulating the damage initiation followed by its propagation without using an initial flaw. However, an explicit crack front is not defined in the cohesive model. In addition, due to the continuum damage description of the cohesive model, an explicit implementation of the fracture mechanics based Paris law cannot be easily accomplished. With predefined surfaces, the strain energy release rate can accurately be computed using a VCCT approach with the knowledge of the crack location or propagation path driven by the self-similar crack growth requirement. An implicit crack front representation based on the extended finite element approach is developed to alleviate the mesh dependence ${ }^{7,8}$.

Given the distinct nature of the modeling approach for static and failure prediction, a unified approach based on a dual spring model is developed for a delamination crack growth prediction. The initiation of a delamination crack under either a static or a peak load prior to the application of fatigue cycles is characterized by a cohesive material model of a user-defined spring element for Abaqus where the damage initiation is driven by the energy dissipation. For a given initial crack under fatigue loading, a distribution of linear penalty springs is used along a pre-defined crack path and the resulting fatigue crack driving force is computed from a VCCT based method by extracting the spring force ahead of the crack front and crack opening displacement behind the front. A Paris law type fatigue growth law with its mode mixity is applied for fatigue crack growth prediction.

The developed dual spring element is verified first using benchmark examples developed by Kruger and Carvalho ${ }^{9-13}$, including Double Cantilever Beam (DCB), End-Notched Flexure (ENF) and Mix-Mode Bending (MMB). The dual spring elements coupled with a continuum damage modeling approach are used next to explore its applicability for the static and fatigue damage prediction of NASA/Boeing sub-elements with the migration of a matrix crack. The effects of mesh sensitivity and a transversely matrix crack parameter on the crack path and crack growth rate behavior are explored during the blind and recalibrated analyses.

Unlike the NASA/Boeing specimen, the applicability of the developed modeling strategy is explored for UTC sub-elements with the presence of multiple ply drop areas. Multiple delamination cracks are simulated at the ply drop locations. While a continuum damage approach is applied for the intra-ply damage description, no material softening from the intra-ply damage is observed. The primary failure model is mainly driven by the propagation of multiple delamination cracks.

\section{A Dual Spring Model}

The dual spring model is developed as a unified approach for static and fatigue failure of laminates interface, including damage initiation and propagation. The dual spring model is implemented via a user-defined spring element for Abaqus, as shown in Fig. 1. The user-defined spring element is used to bond the two corresponding nodal points $\left(P_{1}\right.$ and $\left.P_{2}\right)$ of the two surfaces from the two adjacent plies. The spring behavior is controlled by a penalty stiffness to avoid separation at the intact interface. Under static loading, the failure of the spring is characterized by a cohesive model. It is first linearly loaded up to its failure strength, and further loading damage is initiated until zero load-carrying capacity is reached. The area under the load-deformation curve is the critical energy release rate $G_{c}$. For mixed mode loading conditions, the critical energy release rate follows the Benzeggagh and Kenane (B-K) law,

$$
G_{c}=G_{I c}+\left(G_{I I c}-G_{I c}\right)\left(\frac{G_{\text {shear }}}{G_{T}}\right)^{\eta}
$$

where $G_{I c}, G_{I l c}, G_{\text {shear }}$ and $G_{T}$ are the mode I, mode II, shear and total energy release rate, respectively. $\eta$ is the mode mixity parameter. 


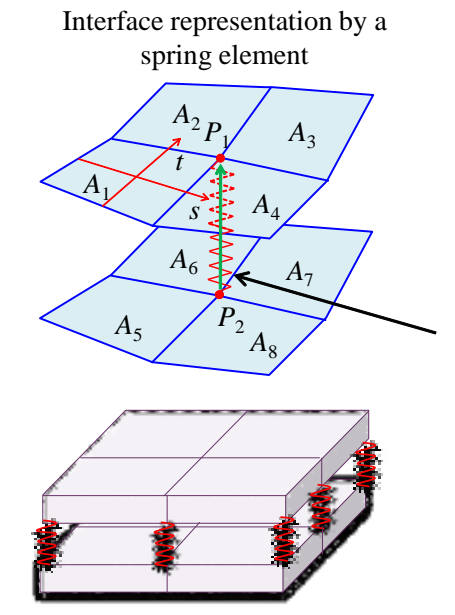

Delamination Characterization

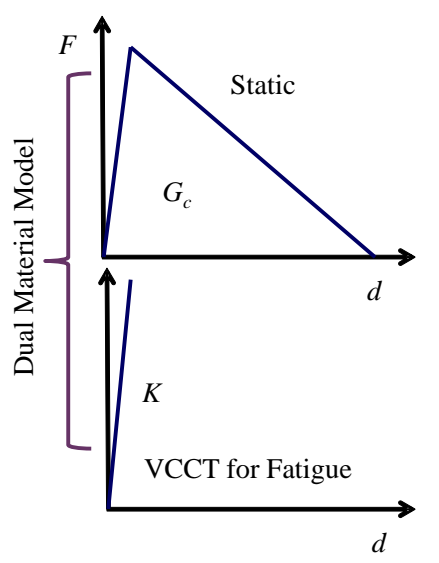

Figure 1. A user-defined spring element in the dual spring model.

Under fatigue loading conditions, the spring is characterized by a linear model and the propagation of the delamination is based on the VCCT technique and Paris law. In order to account for the smoothly distributed traction force at an interface, an effective representative surface area based on the mesh topology is embedded in creation of the user-defined spring element for Abaqus. The mesh independent delamination propagation algorithm under fatigue has been studied by Fang et al. ${ }^{14,15}$. As shown in Fig. 2, the solid and dashed red lines represent the current and propagated delamination fronts, respectively. The delamination front is not conforming to the mesh boundaries. The partially delaminated elements which are cut by the delamination front will be divided into bonded and debonded regions. The elements will be treated as perfectly bonded if less than half of each elemental area gets delaminated, such as those in the light blue zone. Otherwise, they are labeled as fully delaminated as those in the orange colored zone. Then, the boundaries between bonded/debonded elements form a zig-zagged finite element delamination front, denoted by the green solid line in Fig. 2. To apply the VCCT approach, the closing force is extracted from the penalty spring at the nodes on the "FE front" such as node " $P$ ". An associated crack opening sampling point $P^{\prime}$ is taken in the delaminated zone and the line $P P^{\prime}$ ' is vertical to the physical delamination front at point $A$. In the current implementation the length of $P P^{\prime}$ is $0.5 \sqrt{A_{P}}$ where $A_{P}$ is the nodal representative area.

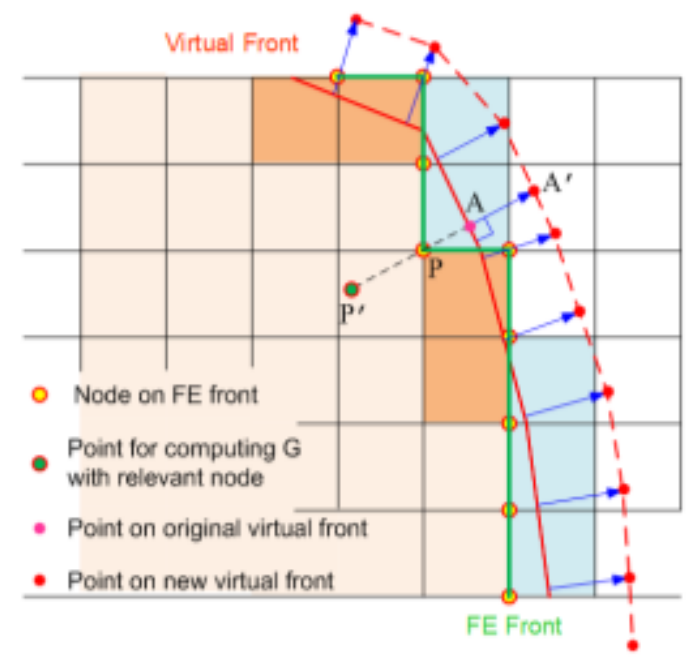

Figure 2. Mesh independent delamination propagation.

Based on VCCT the strain energy release rate $G_{P}$ at the crack opening sampling point $P$ can be computed as 


$$
G_{P}=\frac{1}{2 A_{P}} F_{P} \delta_{P}
$$

where $F_{P}$ is the closing force and $\delta_{P}$ is the relative displacement. The virtual closure area $A_{P}$ is enclosed by the polygon with vertex of displacement sampling point $(P$ '), corresponding "FE front" nodes $(P)$ and two other "FE front" nodes which are adjacent to $P$. The delamination growth rate can be determined by the selected Paris law using the calculated energy release rate. The adopted Paris' Law is given by

$$
\frac{d a}{d N}=c G_{\max }^{m}
$$

where $G_{\max }$ is the strain energy release rate at the maximum fatigue load in one loading cycle. $c$ and $m$ are the Paris' law parameters.

\section{A. DCB}

\section{Verification of Benchmark Examples}

DCB benchmark example is used to verify the performance of our dual spring model under pure mode I failure. The DCB specimen is made of T300/1076 graphite/epoxy with a unidirectional layup, [0] $]_{24}$, and its material properties are listed in Table 1. Mode I fracture toughness $G_{I_{c}}=170.3 \mathrm{~J} / \mathrm{m}^{2}$ and normal tensile strength in matrix direction $Y_{T}=60 \mathrm{MPa}$ are used in the static failure analysis. The geometry and mesh of the DCB model is shown in Fig. 3. The initial crack length is $a=30 \mathrm{~mm}$.

Table 1. Material properties of T300/1076 Unidirectional Graphite/Epoxy Prepreg

\begin{tabular}{|c|c|c|c|c|c|c|}
\hline Properties & $E_{11}(\mathrm{GPa})$ & $E_{22}(\mathrm{GPa})$ & $v_{12}$ & $v_{23}$ & $G_{12}(\mathrm{GPa})$ & $G_{23}(\mathrm{GPa})$ \\
\hline Nominal Value & 139.4 & 10.16 & 0.3 & 0.436 & 4.6 & 3.54 \\
\hline
\end{tabular}
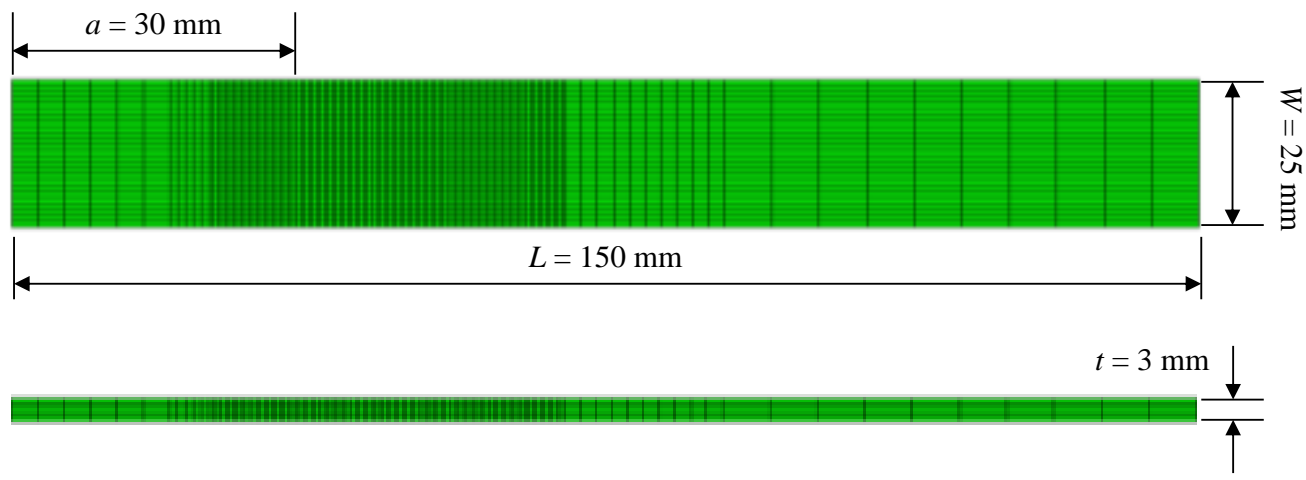

Figure 3. The geometry and mesh of the DCB model.

For static failure analysis, the simulated load vs. displacement curve and crack length vs. displacement curve are compared with the benchmark results ${ }^{9}$, as shown in Fig. 4. It can be seen that the simulated results agree well with the benchmark results for DCB static failure analysis. 


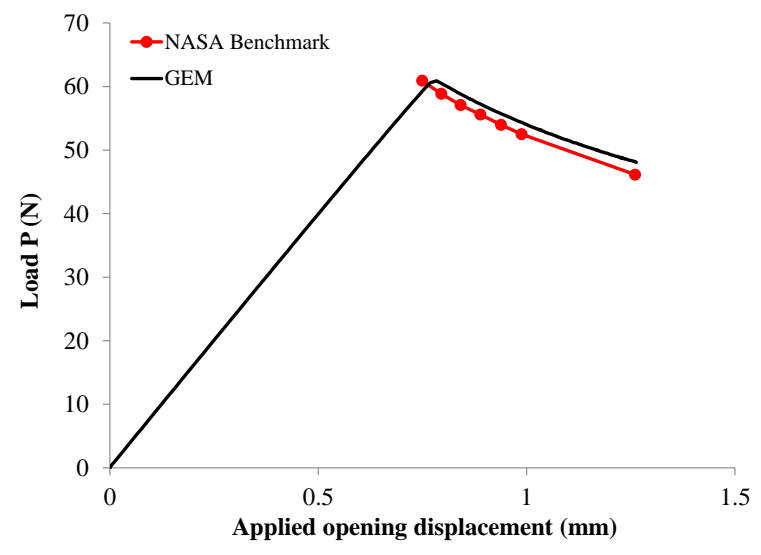

(a)

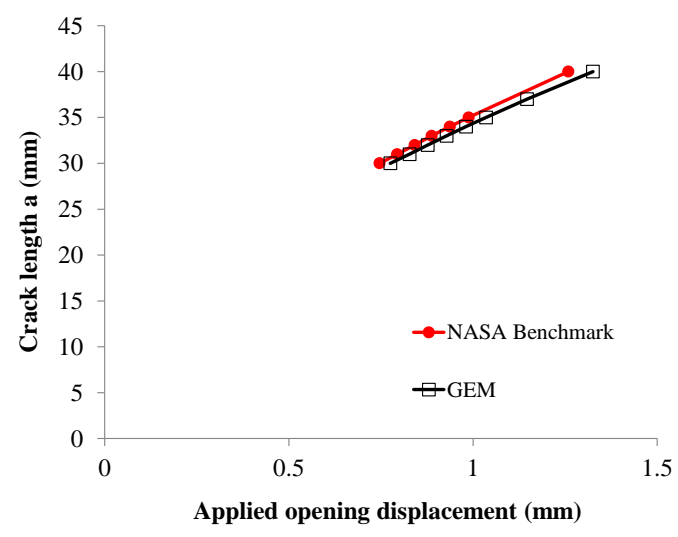

(b)

Figure 4. The comparison of simulation results with benchmark results: (a) Load vs. displacement curve; (b) Crack length vs. displacement curve.

In the fatigue analysis, the same finite element model is adopted. The Paris Law parameters are $c=2.44 \mathrm{e} 6$ and $n$ $=10.61$, and the delamination growth onset parameters are $m_{0}=0.2023$ and $m_{1}=-0.078924$. The simulated crack length vs. cycles curve is compared with the benchmark results ${ }^{10}$, as shown in Fig. 5 . It can be seen that the simulated results also agree well with the benchmark results for DCB fatigue analysis.

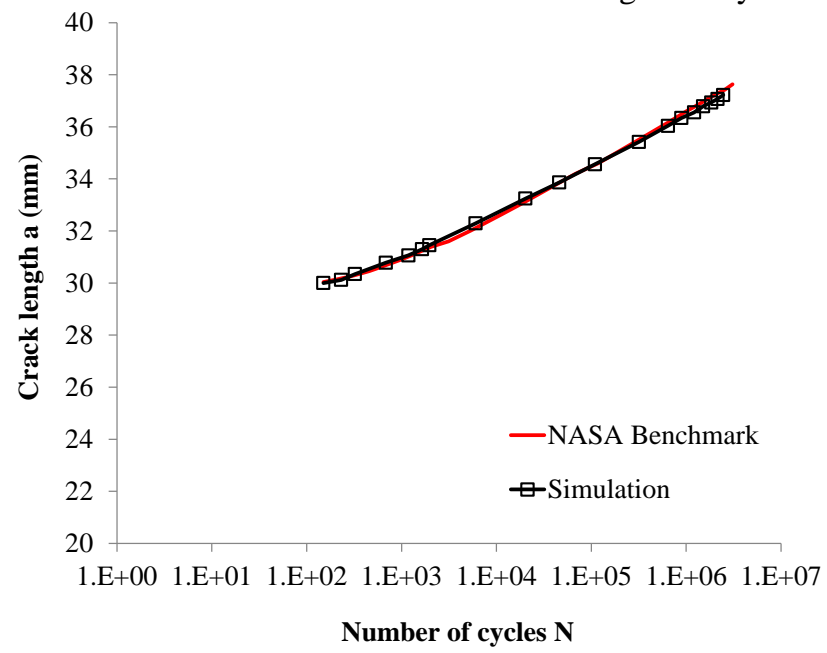

Figure 5. The comparison of simulated crack length vs. cycles curve with benchmark result.

\section{B. ENF}

ENF benchmark example is used to verify the performance of our dual spring model under pure mode II failure. The ENF specimen is made of IM7/8552 graphite/epoxy with a unidirectional layup, $[0]_{24}$, and its material properties are listed in Table 2. Mode II fracture toughness $G_{I I c}=780 \mathrm{~J} / \mathrm{m}^{2}$ and shear strength $S=95.84 \mathrm{MPa}$ are used in the static failure analysis. The geometry and mesh of the ENF model is shown in Fig. 6. The initial crack length is a $=25.4 \mathrm{~mm}$.

Table 2. Material properties of IM7/8552 Unidirectional Graphite/Epoxy Prepreg

\begin{tabular}{|c|c|c|c|c|c|c|}
\hline Properties & $E_{11}(\mathrm{GPa})$ & $E_{22}(\mathrm{GPa})$ & $v_{12}$ & $v_{23}$ & $G_{12}(\mathrm{GPa})$ & $G_{23}(\mathrm{GPa})$ \\
\hline Nominal Value & 161 & 11.38 & 0.32 & 0.45 & 5.2 & 3.9 \\
\hline
\end{tabular}



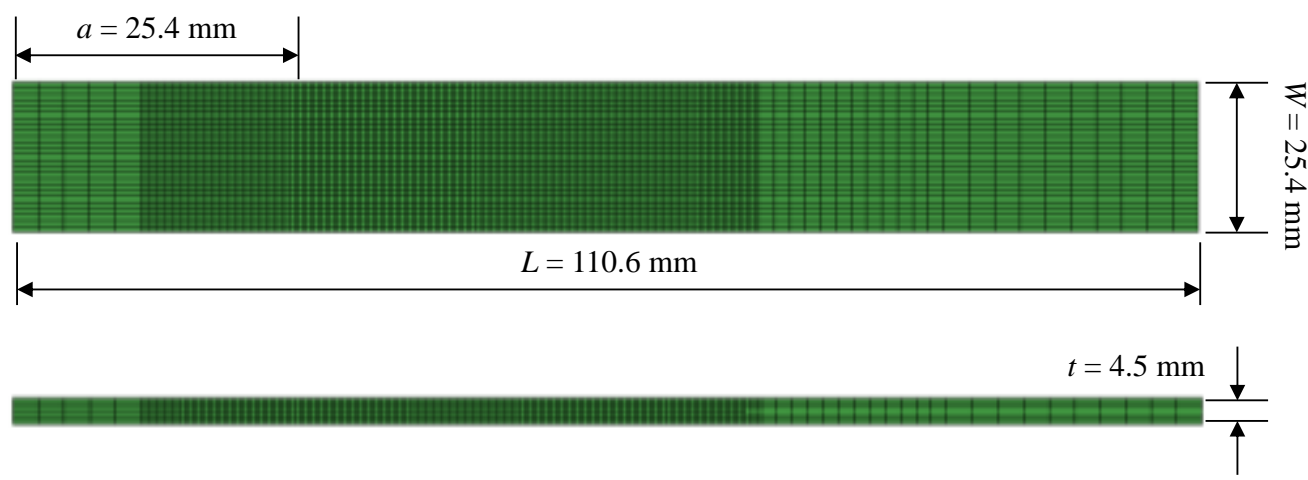

Figure 6. The geometry and mesh of the ENF model.

For static failure analysis, the simulated load vs. displacement curve and displacement vs. increase in delamination length curve are compared with the benchmark results ${ }^{11}$, as shown in Fig. 7. It can be seen that the simulated results agree well with the benchmark results for ENF static failure analysis.

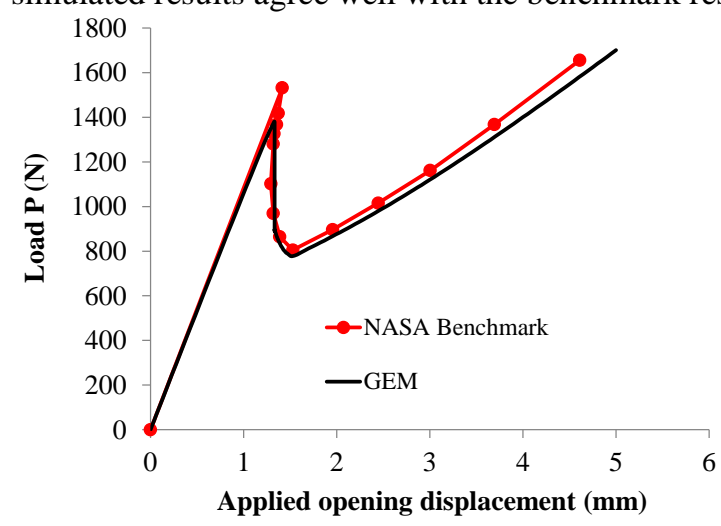

(a)

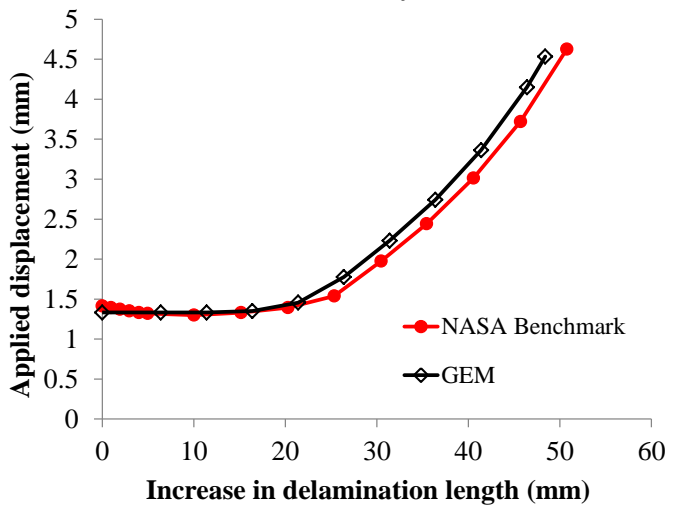

(b)

Figure 7. The comparison of simulation results with benchmark results: (a) Load vs. displacement curve; (b) Displacement vs. increase in delamination length curve.

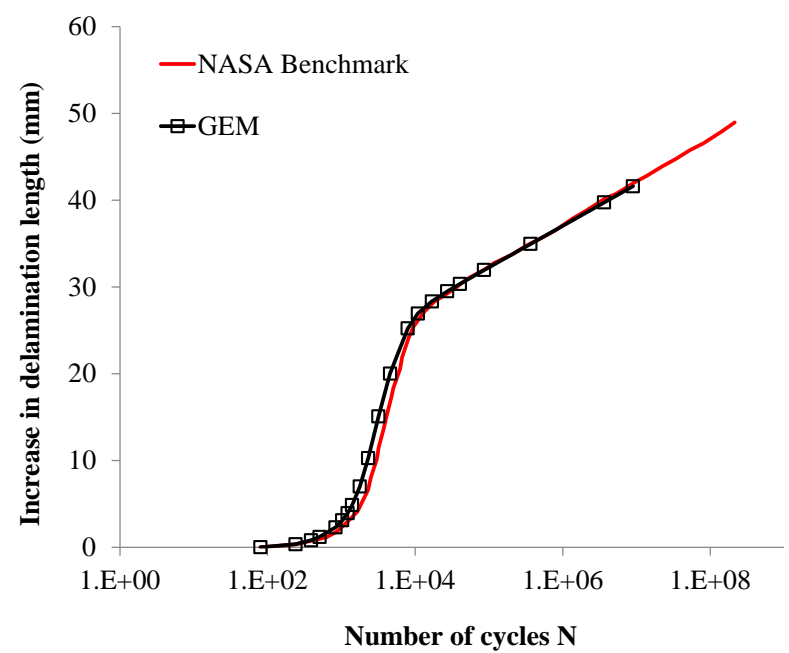

Figure 8. The comparison of simulated increase in delamination length vs. cycles curve with benchmark result.

In the fatigue analysis, the same finite element model is adopted. The Paris Law parameters are $c=0.33$ and $n=$ 5.55 , and the delamination growth onset parameters are $m_{0}=0.78$ and $m_{1}=-0.16$. The simulated crack length vs. 
cycles curve is compared with the benchmark results ${ }^{11}$, as shown in Fig. 8. It can be seen that the simulated results also agree well with the benchmark results for ENF fatigue analysis.

\section{MMB}

MMB benchmark example is used to verify the performance of our dual spring model under mix-mode failure. The MMB specimen is made of IM7/8552 graphite/epoxy with a unidirectional layup, $[0]_{24}$, and its material properties are listed in Table 2. The fracture toughness $G_{I c}=0.212 \mathrm{~kJ} / \mathrm{m}^{2}$ and $G_{I I c}=0.774 \mathrm{~kJ} / \mathrm{m}^{2}$, normal tensile strength in matrix direction $Y_{T}=115.83 \mathrm{MPa}$ and strength shear strength $S=95.84 \mathrm{MPa}$ are used in the static failure analysis. The geometry and mesh of the MMB model is shown in Fig. 9. The initial crack length is $a=25.4 \mathrm{~mm}$. One case with mix mode ratio $G_{I I} / G_{T}=0.2$ is simulated.

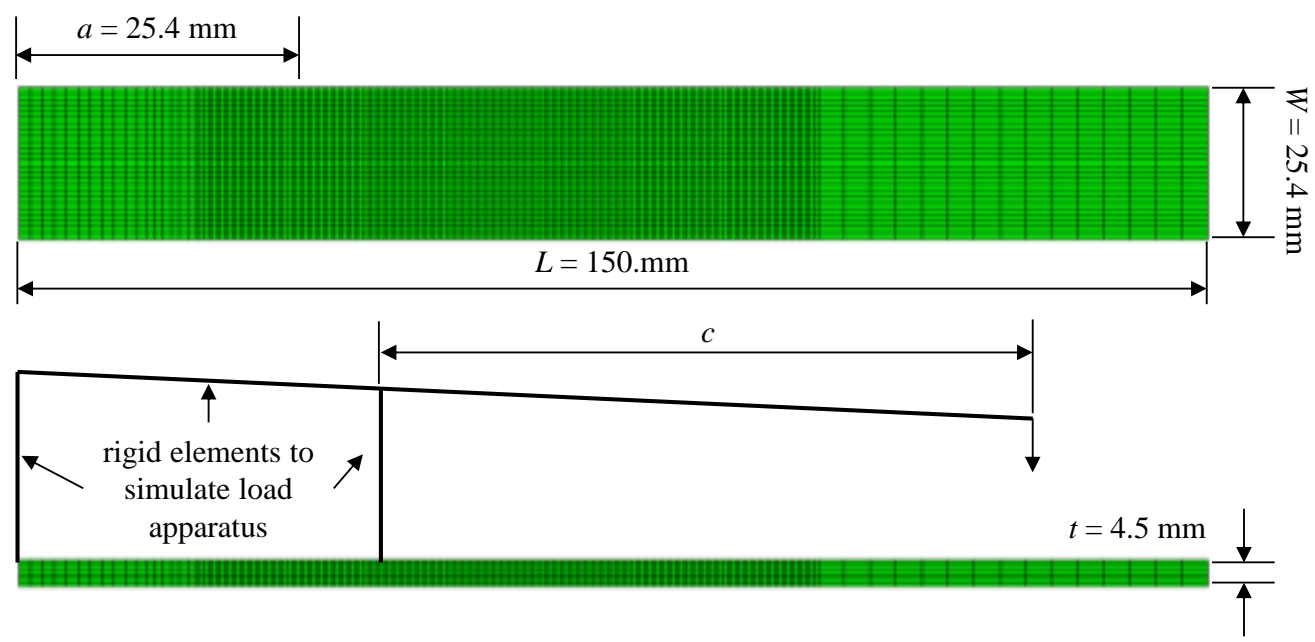

Figure 9. The geometry and mesh of the MMB model.

For static failure analysis, the simulated load vs. displacement curve and displacement vs. increase in delamination length curve are compared with the benchmark results ${ }^{12}$, as shown in Fig. 10. It can be seen that the simulated results agree well with the benchmark results for MMB static failure analysis.

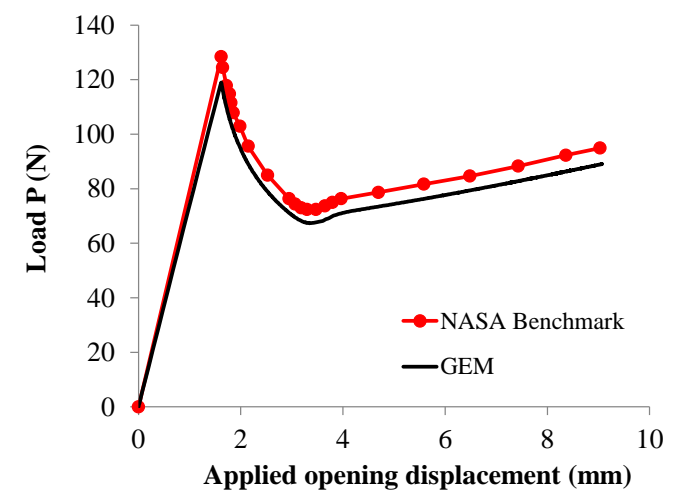

(a)

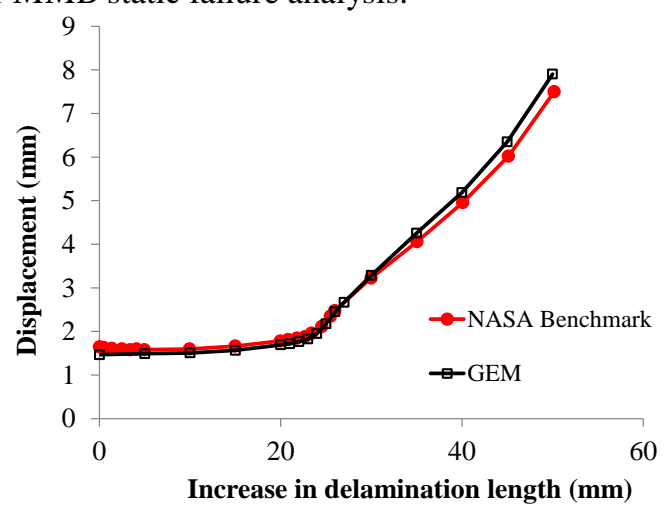

(b)

Figure 10. The comparison of simulation results with benchmark results for $G_{I I} / G_{T}=0.2$ : (a) Load vs. displacement curve; (b) Displacement vs. increase in delamination length curve.

In the fatigue analysis, the same finite element model is adopted. The Paris Law parameters are $c=2412$ and $n=$ 8.4 , and the delamination growth onset parameters are $m_{0}=0.304$ and $m_{1}=-0.09$. The simulated crack length vs. cycles curve is compared with the benchmark results ${ }^{13}$, as shown in Fig. 11. It can be seen that the simulated results also agree well with the benchmark results for MMB fatigue analysis. 


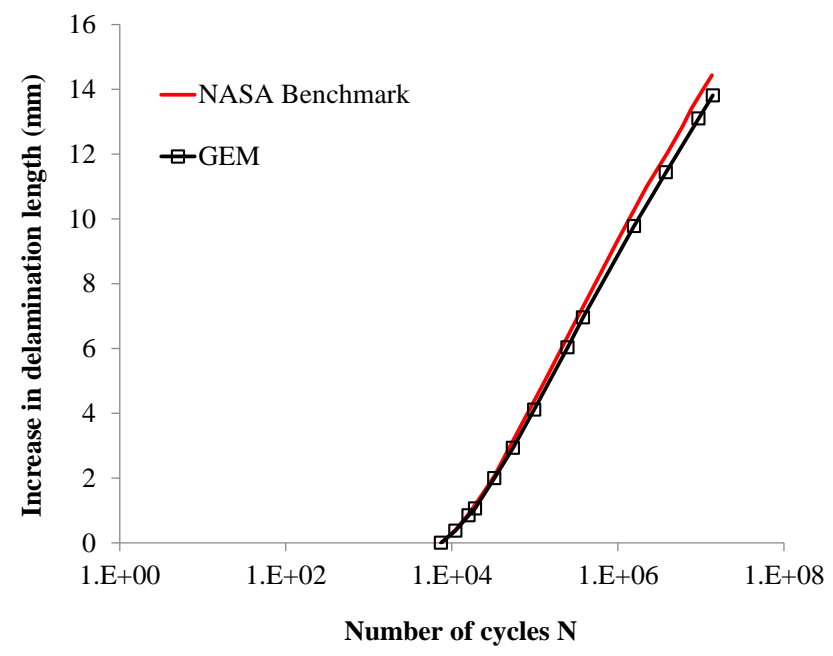

Figure 11. The comparison of simulated increase in delamination length vs. cycles curve with benchmark result for $G_{I I} / G_{T}=0.2$.

\section{Static and Fatigue Damage Assessment of Carbon/Epoxy Crack Migration Sub-element}

The dual spring model briefly discussed above is applied to the static and fatigue failure prediction of a carbon/epoxy sub-element, which involves matrix cracking and delamination initiation, propagation and migration. Hereinafter, this design is referred to as the 'NASA/Boeing sub-element.' The finite element model including the loading fixture is shown in Fig. 2. The composite specimen is clamped by two fixing blocks and the base steel plate, which is fixed at the bottom. The finite element model has only one element of 0.01 inch in the width direction normal to the plane of the paper. The degree of freedom in the out of plane, i.e. width direction of all the parts, is fixed to simulate the plane strain condition. Periodic boundary conditions are applied in the remaining two degrees of freedom for the test article. Two interfaces between 0/90 plies are modeled using Abaqus cohesive elements, which are marked in red. Four 90 plies between these two interfaces are simulated using continuum damage mechanics (CDM) model ${ }^{16}$, which are marked in blue. The rest of the part is characterized using Abaqus C3D8I elements, which are marked in grey. The load point shown in Fig. 12 is associated with Configuration A. Configuration B is created by moving the load hinge to the left.

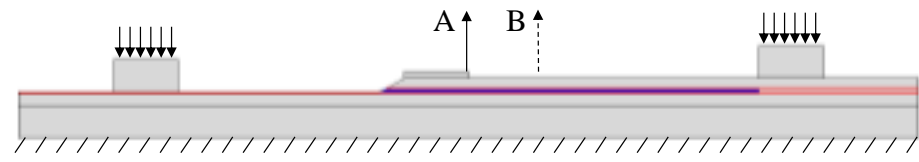

Figure 12. The finite element model of NASA sub-element for static analysis.

The material properties of IM7/8552 for static and fatigue analysis can be found in literatures ${ }^{17-20}$.

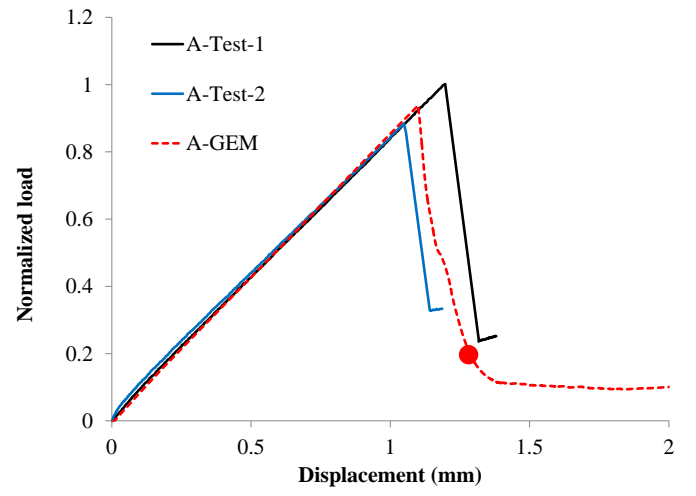

(a)

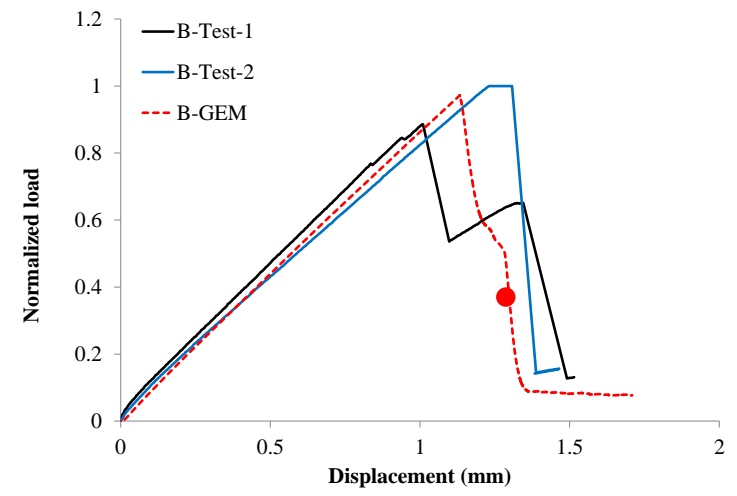

(b)

Figure 13. Comparison of load displacement curves between the prediction and the test data (a) Configuration A; (b) Configuration B.

8

American Institute of Aeronautics and Astronautics 


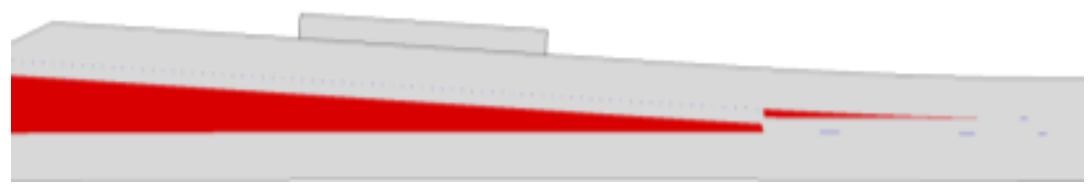

Figure 4. Migration in the NASA sub-element for Configuration B.

The predicted load displacement curves are compared with the test data for Configuration A and B, as shown in Fig. 13. The load is normalized using the peak load from test data. The predicted peak loads agree well with the test data. Same as in the experiment, the load displacement curves are almost linear before reaching the peak load. A quick drop of the load is observed after the peak followed by a segment of a slightly reduced rate of load drop. The load drop changing point is not very clear for Configuration A, which is not observed from the experiment. For Configuration $\mathrm{B}$, the load changing point can be considered as the first arrest point, which is observed in one of the testing results. There is an obvious arrest when the load drops to about 0.2 of the peak load in the test data of Configuration A. For Configuration B, the predicted seconded arrest point is very close to the test data. The migration occurs in the fast load drop region before this arrest point for both Configuration A and B and the migration points are highlighted in Fig. 13. The migration in Configuration B is shown in Fig. 14.

In the fatigue simulation, the crack path is predefined based on the static prediction, as shown in Fig. 15. Two different load levels are used in the fatigue test with load ratio of 0.1 and only Configuration A is studied in the fatigue analysis.

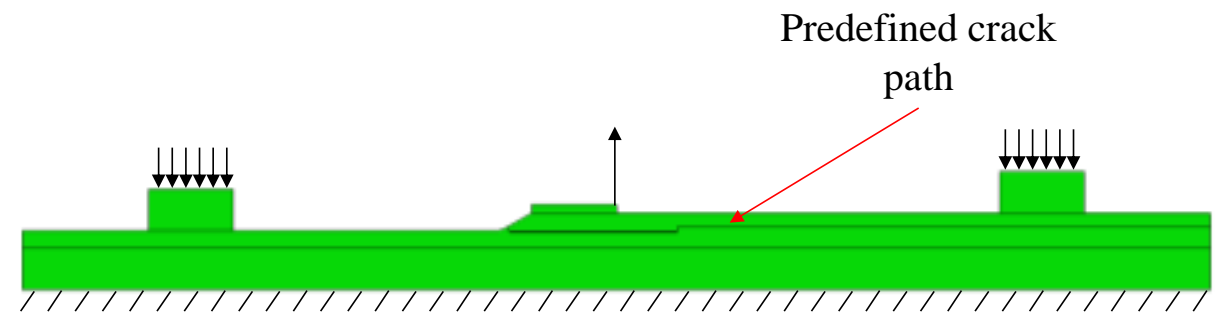

Figure 15. The finite element model of NASA sub-element for fatigue analysis.

The predicted delamination length vs. fatigue cycles curve is compared with the test data, as shown in Fig. 16. The predicted fatigue cycle number agrees well with the test data at the initial stage, while the subsequent predicted delamination growth occurs at a faster rate than that observed in the experiments. 

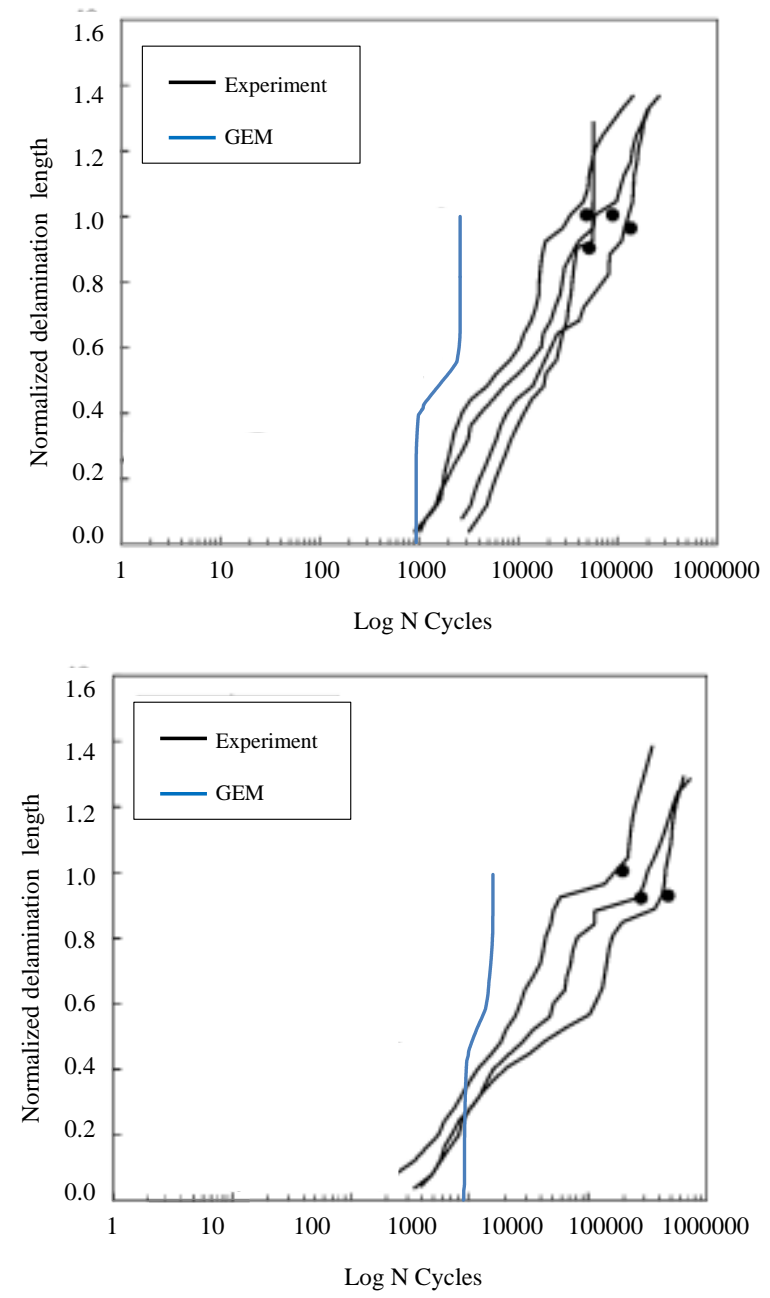

(a)

(b)

Figure 16. The finite element model of NASA sub-element for static analysis: (a) High fatigue load; (b) Low fatigue load.

\section{Static and Fatigue Damage Assessment of Carbon/Epoxy Sub-element with Manufacturing Discontinuities}

The dual spring model developed is utilized next for the static and fatigue failure prediction of another carbon/epoxy sub-element, which involves delamination initiation and propagation at ply drop locations. Hereinafter, this design is referred to as the 'UTC sub-element'. The loading configuration of the UTC sub-element is shown in Fig. 17 and different loading configurations are studied by changing the support span $L_{s}$. The composite specimen has 50 zero-degree plies and wrapped with a laminate of [0] layup. The models developed in this study do not account for inconsistencies observed in the as-fabricated sub-element including variations in spatial thickness and the size, shape and location of ply-drops. The material properties are the same as those used in the NASA/Boeing sub-element model. 


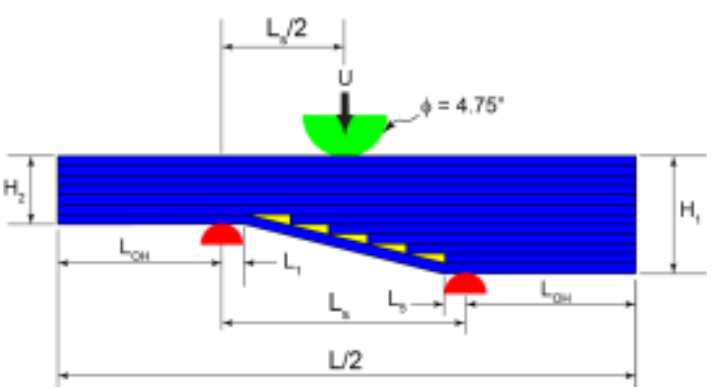

Figure 17. The loading configuration of UTC sub-element test.

A parametric study is performed in the static prediction by using two different values of mode II fracture toughness of $4.22 \mathrm{psi}$ in and $6.5 \mathrm{psi}$ in associated with pre-crack and non-pre-crack ENF test coupons, respectively. Nonlinear shear behavior of the composite laminates is characterized within the CDM model and the difference in ply stiffness under longitudinal tension and compression is also considered. A comparison of the normalized load displacement curves is shown in Fig. 18 and 19 for the two testing configurations. In Configuration \# 1, $L_{s}=3.0$ in., whereas in Configuration \# 2, $L_{s}=3.25$ in. It can be seen that the predicted peak load agrees well with the test data. The predicted peak load using a smaller $G_{I I C}$ is lower than the one using the higher $G_{I I C}$.

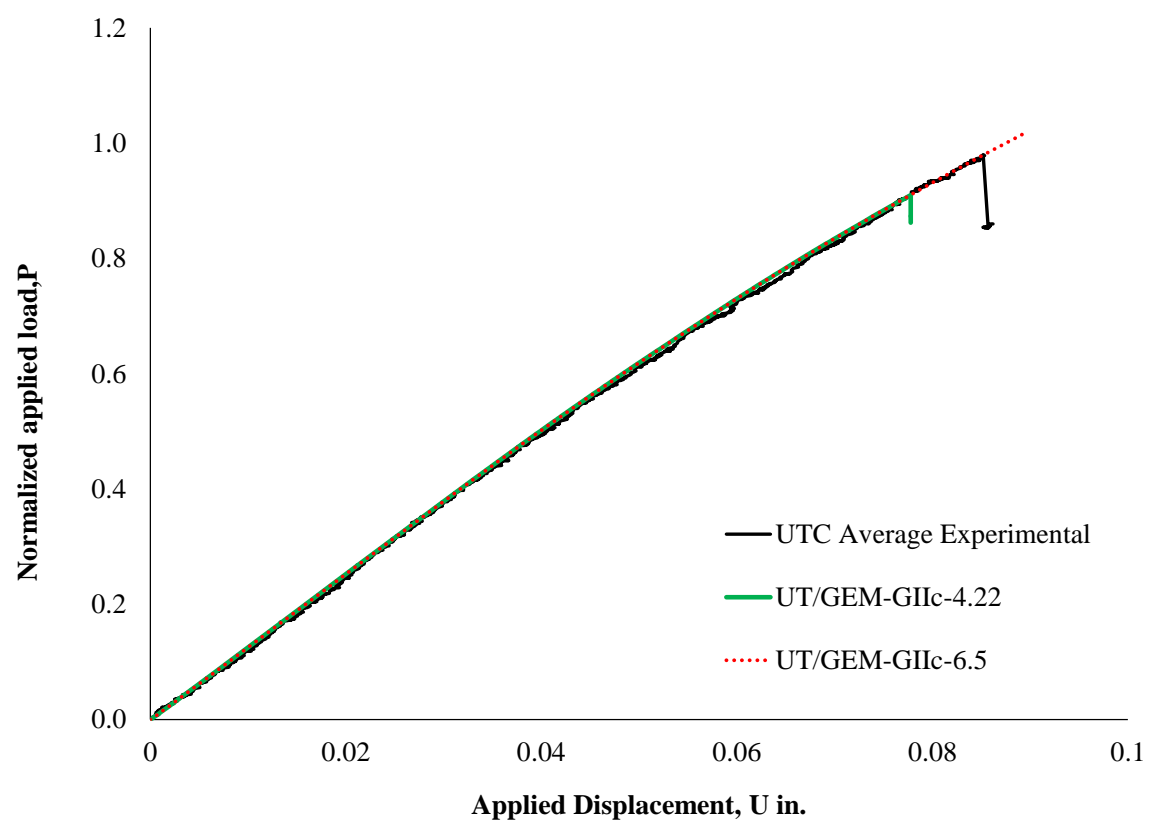

Figure 18. The comparison of load vs. displacement curve of blind and calibrated prediction with the test data for Configuration \#1. 


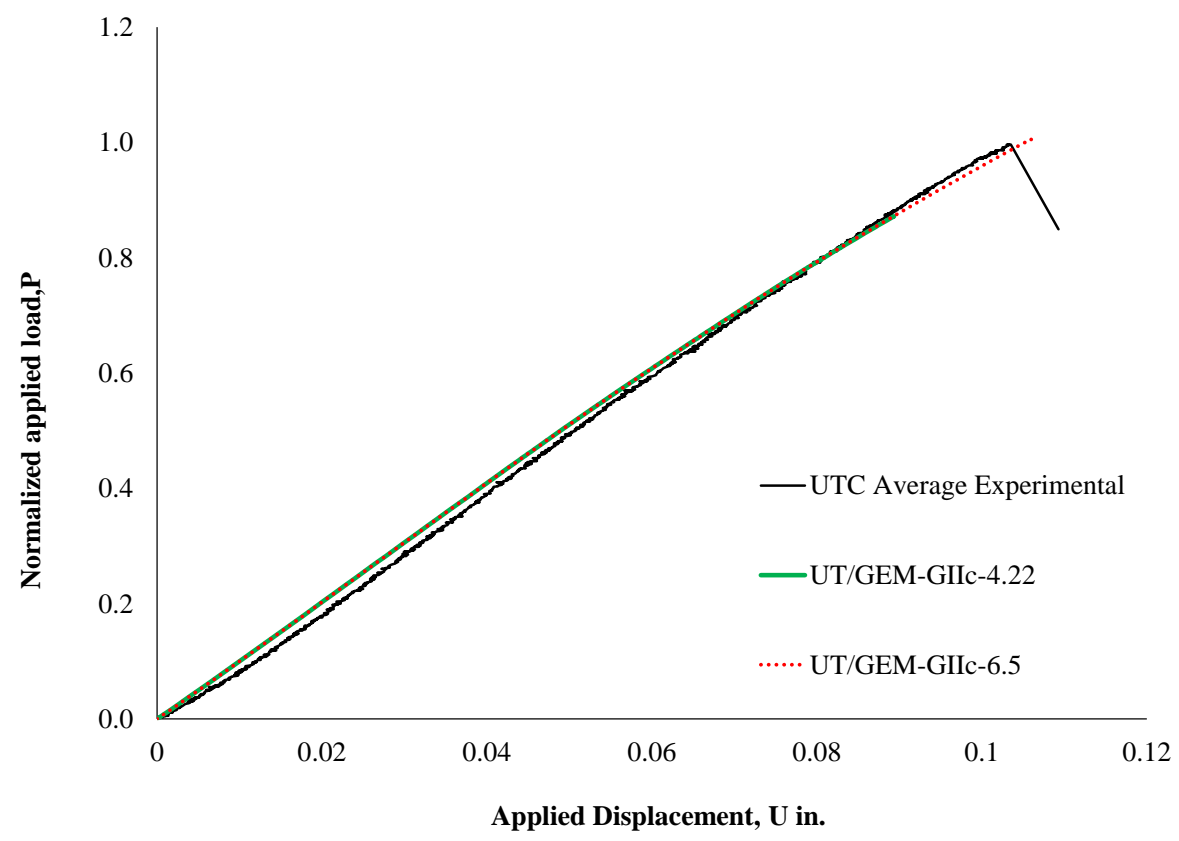

Figure 19. The comparison of load vs. displacement curve of blind and calibrated prediction with the test data for Configuration \#2.

Same as in the prediction of the NASA/Boeing sub-element, a crack path is predefined according to the static prediction, which is near one of the resin rich regions. The predicted load vs cycle curve is compared with the test data. Unlike the NASA/Boeing sub-element simulation, the effect of including nonlinear shear is quite significant in the fatigue simulation of the UTC sub-element. The predicted load vs cycle curve is compared with the test data, as shown in Fig. 20. Without nonlinear shear, the fatigue load is over-predicted perhaps because the models are based on the ideal design geometry. However, the total cycles to failure is predicted to be within the experimental scatter. With non-linear shear, the predictions of fatigue load carrying capacity exhibit better agreement with experiments, perhaps because of the additional compliance of the sub-element. However, in this case, the total cycles to failure increase perceptibly. Therefore, when comparing the static and fatigue modeling results, it may be argued that there is interplay between the geometric and material parameters which critically influences the predictions.

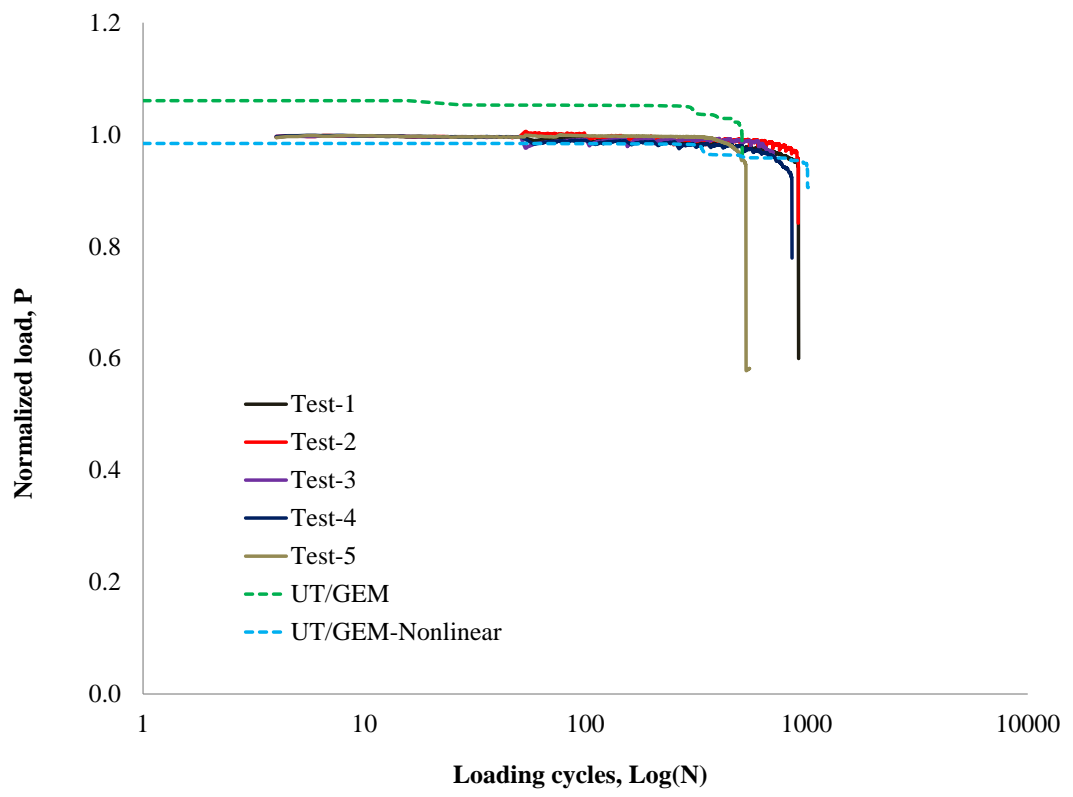

Figure 20. The comparison of predicted load vs. cycle curve with the test data.

American Institute of Aeronautics and Astronautics 


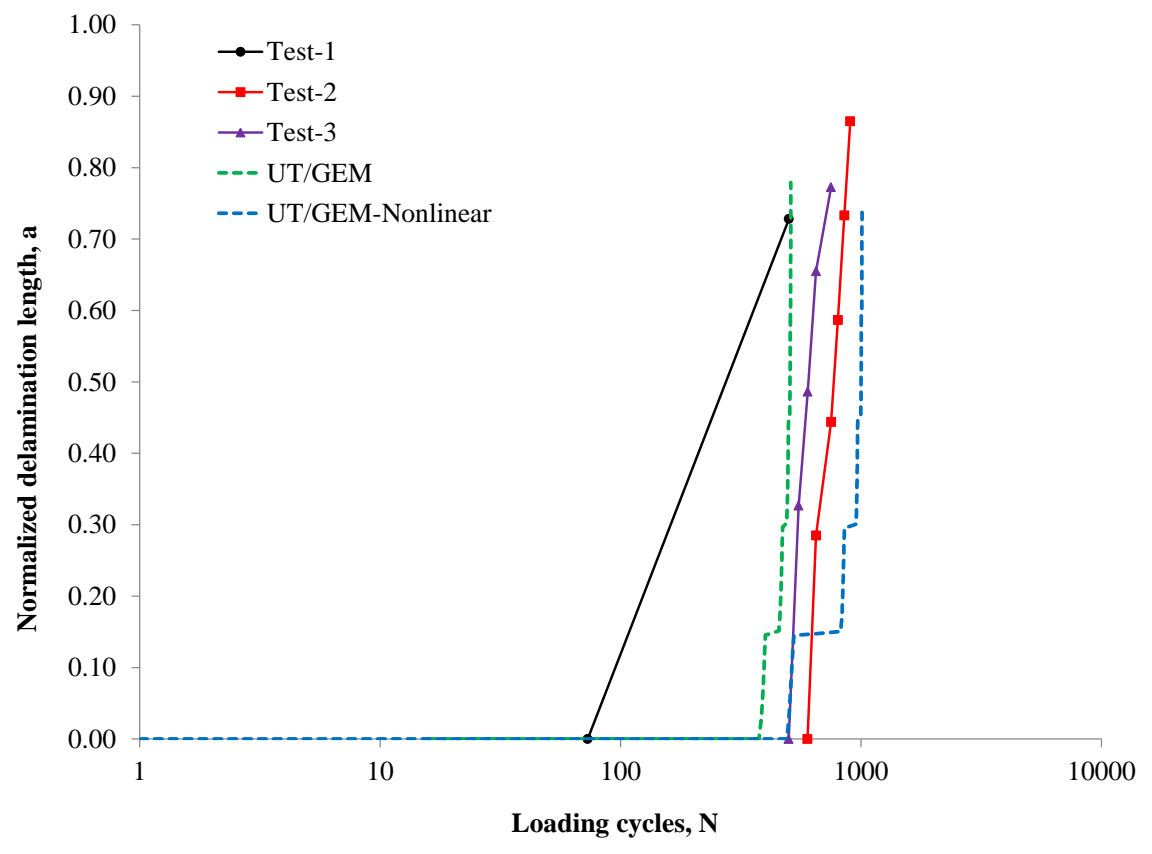

Figure 21. The comparison of predicted crack length vs. cycle curve with the test data.

The predicted crack length vs. cycle curve is compared with the test data, as shown in Fig. 21. The crack length measured is the total length of the major crack, which is the crack along the inclined interface. It can be seen that the predicted delamination growth with fatigue cycles agrees quite well with experimental data. After including the nonlinear shear into the fatigue model, a slower delamination growth is observed.

\section{Discussion}

In the verification of the dual spring model with DCB, ENF and MMB benchmark examples, the simulation results agree well with the benchmark results in static and fatigue analysis. It should be noted that the predicted peak load is always slightly lower, which is due to the intrinsic behavior of the cohesive model with its finite initial stiffness. A large penalty stiffness has to be selected adequately to balance the accuracy and numerical stability. Especially for the ENF case, the results have exhibited an unstable behavior where its critical displacement decreases at the beginning of the load drop. This unstable delamination propagation cannot be captured even using a displacement controlled loading. Viscous regulation has to be introduced to remedy the convergence issue.

From static prediction of NASA/Boeing testing case, the migration associated with the matrix cracking within the four 90-ply can be captured using the CDM approach with the energy driven progressive damage.

In the fatigue analysis, an initial crack of several element sizes has to be introduced for the extraction of the energy release rate. An S-N based crack initiation module can be employed with a calibration to account for the presence of stress concentration. Conservative fatigue life predictions for the NASA/Boeing sub-element indicate that the use of the point stress based crack initiation followed by the $G(N)$ based onset life prediction may not be accurate for the component with the presence of the stress concentration. In addition, the inaccurate crack propagation life prediction could be due to the lack of data to account for the R-ratio dependent fatigue behavior.

\section{Conclusion}

A dual spring model has been developed to perform both static and fatigue prediction of laminates. Within the computational framework of the dual spring model, the static failure along with its damage progression has been performed via the cohesive characteristic of the spring while the fatigue failure has been captured using the penalty characteristic of the spring. Verifications using benchmark examples, including DCB, ENF, and MMB specimens have demonstrated both the accuracy and numerical stability of the dual spring model. Static and fatigue predictions have been performed for NASA/Boeing and UTC sub-element. 
For the NASA/Boeing sub-element, the onset and arrest behavior of crack growth has been captured for the two configurations. The predicted peak load and subsequent failure modes agree very well with the test data. The simulation of static test of the UTC sub-element has been performed at two different values of supporter (roller) distance. Good agreement between predictions and the test data is also achieved for the initial stiffness and the peak load.

The predicted fatigue lives for the NASA/Boeing sub-element are conservative. The use of the point stress based crack initiation followed by the $\mathrm{G}(\mathrm{N})$ based onset life prediction may not be a rational approach for the component with stress concentration. The inaccurate crack propagation life prediction could be due to the inaccuracy of the Rratio dependent fatigue data. The predicted fatigue load drop and crack length growth agree well with the test data for UTC sub-element. Different from the NASA/Boeing sub-element, the UTC sub-element is driven by a Mode II dominated fatigue failure. Same as static analysis, nonlinear shear has substantial effect on the fatigue failure development.

\section{Acknowledgments}

The authors from Global Engineering and Materials, Inc. are grateful for the sub-contract award from United Technologies Corporation (UTC). UTC was funded by NASA under Award No. NNL09AA00A.

\section{References}

${ }^{1}$ Turon, A., Costa, J., Camanho, P.P., and Dávila, C.G., "Simulation of delamination in composites under high-cycle fatigue," Composites Part A: Applied Science and Manufacturing, Vol. 38, No. 11, 2007, pp. 2270-2282.

${ }^{2}$ May, M., and Hallett, S.R., "A combined model for initiation and propagation of damage under fatigue loading for cohesive interface elements," Composites Part A: Applied Science and Manufacturing, Vol. 41, No. 12, 2010, pp. 1787-1796.

${ }^{3}$ May, M., and Hallett, S.R., "An advanced model for initiation and propagation of damage under fatigue loading - part I: Model formulation," Composite Structures, Vol. 93, No. 9, 2011, pp. 2340-2349.

${ }^{4}$ Hoos, K., Iarve, E.V., Braginsky, M., Zhou, E., and Mollenhauer, D.H.,. "Progressive Failure Simulation in Laminated Composites under Fatigue Loading by Using Discrete Damage Modeling," 57th AIAA/ASCE/AHS/ASC Structures, Structural Dynamics, and Materials Conference, San Diego, CA, 2016.

${ }^{5}$ Wimmer, G., Schuecker, C., and Pettermann, H. E., "Numerical simulation of delamination onset and growth in laminated composites," Austrian Aeronautics Research, Network for Materials and Engineering, ILDSB, Vienna University of Technology, 2006.

${ }^{6}$ Krueger, R., "Virtual crack closure technique: history, approach, and applications," Applied Mechanics Reviews, Vol. 57, No. 2, 2004, pp. 109-143.

${ }^{7}$ Latifi, M., van der Meer, F.P., and Sluys, L.J., “A level set model for simulating fatigue-driven delamination in composites,” International Journal of Fatigue, Vol. 80, 2015, pp. 434-442.

${ }^{8}$ Grogan, D.M., Leen, S.B., and Ó Brádaigh, C.M., "An XFEM-based methodology for fatigue delamination and permeability of composites," Composite Structures, Vol. 107, 2014, pp. 205-218.

${ }^{9}$ Krueger, R., "An approach to assess delamination propagation simulation capabilities in commercial finite element codes," NASA TM-2008-215123, 2008.

${ }^{10}$ Krueger, R., "Development of a benchmark example for delamination fatigue growth prediction," NASA CR-2010-216723, 2010 .

${ }^{11}$ Krueger, R., "Development and application of benchmark examples for mode II static delamination propagation and fatigue growth predictions," NASA CR-2011-217305, 2011.

${ }^{12}$ Krueger, R., "Development and application of benchmark examples for mixed-mode I/II quasi-static delamination propagation predictions," NASA CR-2012-217562, 2012.

${ }^{13}$ Krueger, R., and Carvalho, N., "In Search of a Time Efficient Approach to Crack and Delamination Growth Predictions in Composites," 31st ASC Technical Conference, Williamsburg, VA, 2016.

${ }^{14}$ Fang, E., Stuebner, M., and Lua, J., "X-FEM Co-Simulation of Delamination and Matrix Cracking in Fiber Metal Laminated Structures under Fatigue Loading," 54th AIAA/ASME/ASCE/AHS/ASC Structures, Structural Dynamics, and Materials Conference, Structures, Structural Dynamics, and Materials Conferences, Boston, MA, 2013.

${ }^{15}$ Fang, E., Cui, X., and Lua, J., "An Integrated Crack Initiation and Propagation Module for Fatigue Damage Prediction of Composite Structures," 58th AIAA/ASCE/AHS/ASC Structures, Structural Dynamics, and Materials Conference, Grapevine, Texas, 2017.

${ }^{16}$ Fang, E., Cui, X., and Lua, J., "Comparative Composite Fatigue Damage Models for Life Prediction of Laminated Composite Structures," 30th ASC Technical Conference, East Lansing, MI, 2015. 
${ }^{17}$ Makeev, A., Seon, G., Nikishkov, Y., Nguyen, D., Mathews, P. and Robeson, M., “Analysis Methods Improving Confidence in Material Qualification for Laminated Composites," AHS 72nd Annual Forum, West Palm Beach, FL, 2016, pp. 1719.

${ }^{18} \mathrm{O}$ 'Brien, T.K., Johnston, W.M., and Toland, G.J., "Mode II interlaminar fracture toughness and fatigue characterization of a graphite epoxy composite material," NASA TM-2010-216838, 2010.

${ }^{19}$ Murri, G.B., "Evaluation of delamination onset and growth characterization methods under mode I fatigue loading," NASA TM-2013-21766, 2013.

${ }^{20}$ Ratcliffe, J.G., and Johnston Jr, W.M., "Influence of mixed mode I-mode II loading on fatigue delamination growth characteristics of a graphite epoxy tape laminate," 29th ASC Technical Conference, San Diego, CA, 2014. 